\title{
Getreide, Getreideerzeugnisse u. dgl.
}

\section{Brot und Backwaren}

E. Villegas, Y.Pomeranz und J. A. Shellenherger: Einflïsse sulfhydroxylierter Gelatinepräparate und von Glutathion auf die rheologisehen Eigenschaften von Weizenteigen. (Effects of thiolated gelatins and glutathione on rheological properties of wheat doughs.) (Manhattan, Kans., Crops Res. Div., Agric. Res. Serv., U.S. Dept. of Agric., and Dept. of Flour and Feed Milling Industr., Kansas State Univ.) Cereal Chem. 4.0, $694-703$ (1963).

Zusatz von 3 sulfhydroxylierten Gelatinepräparaten mit 14,2, 13,3 und 3,2 Val-sH in $100 \mathrm{~kg}$ in Mengen von $0,5 \%$ und von Glutathion zu Mehlen bewirkte bei der Teigbereitung ein leichtes Absinken der farinographisch gemessenen Wasseraufnahme und setzte die Teigentwicklungszeit sowie den Valorimeterwert beträchtlich herab. Die Dehnbarkeit der Teige wird durch Thiogelatine und Glutathion vergrößert, die Elastizität herabgesetzt; die Extensigrammflächen werden verkleinert, wobei Glutathion den stärksten Einfluß hat. Die durch Thiogelatine bewirkten Änderungen der rheologischen Teigeigenschaften können durch einen Überschuß an Oxydantien (z.B.das Dreifache der dem SH-Gehalt äquivalenten Menge an $\mathrm{KBrO}_{3}$ ) oder von $\mathrm{N}$ - $\mathrm{A}$ thylmaleinimid (NEMI) teilweise oder ganz aufgehoben werden, jedoch nicht die durch Glutathion bewirkten Änderungen. Glutathion und Thiogelatine verstärken das Weichwerden der Teige bei mechanischem Bearbeiten; gleichzeitiger Zusatz von NEMI hebt den Einfluß auf.

Glutathion und größere Zusätze von Thiogelatine $(0,33-0,35 \mu \mathrm{mol}$ SH je g) Mehl verringern das Gebäckvolumen merklich und verschlechtern die übrigen Brotwertmerkmale, jedoch ist der Grad des Einflusses von der Herkunft der Mehle abhängig, wie vergleichende Versuche mit Mehlen aus verschiedenen Getreidesorten zeigten.

W. ERner (Berlin)

G. R. Jackson und B. W. Landfried: Der Einfluß verschiedener Glyceride auf die Backeigenschaiten von Stärketeigen. (The effect of various glycerides on the baking properties of starch doughs.) (Dolton, Ill., Dolton Manufacturing Co., und Kansas City, Kans., Breddo Food Products Corp.) Cereal Chem. 42, 323-330 (1965).

In Fortsetzung und Ergänzung der Untersuchung von JoNGH (1961) über die strukturbildenden Figenschaften der Stärke in Gegenwart von Glycerinmonostearat sind Versuche mit Weizenstärketeigen unter Zusatz von Mono-, Di- und Triglyceriden unternommen worden. Das Verhalten der mit Hefe gelockerten Stärketeige zeigt sich an der Krumenstrucktur daraus hergestellter Gebäcke schon bei Zusätzen von 0,1\%. Dabei ist der Grad der Sättigung wesentlicher als das Verhältnis von Mono- zu Diglyceriden. Total gesättigte Glyceride sind wirksamer in bezug auf die gewünschte Struktur.

M. Rohrlich (Berlin)

K. Kismarton: Beitrag zur rheologischen Charakterisierung des Brotes. [Ungarisch mit dtsch., engl., französ. und russ. Zusammenfass.] (Miskolc, Megyei Minöségvizsgáló Intézet.) Élelmiszervizsgálati Közlemények 11, 57-61 (1965).

Verf. arbeitete eine die rheologische Konsistenz der Brotkrume mit einem einzigen Wert kennzeichnende Indexzahl aus. Aus den Meßergebnissen der gesamten und der elastischen Formänderung lassen sich die plastische (bleibende) Formänderung und die relative Elastizität berechnen. Aus diesen Angaben bildete der Verf. seine ,rheologische Indexzahl".

$$
\text { J. Kottász und I. Gál (Budapest) }
$$

G. Rubenthaler, Y. Pomeranz und K. F. Finney: Die Wirkung von Zuckern und bestimmten freien Aminosänren auf Characteristica des Brotes. (Effects of sugars and certain free amino acids on bread characteristics.) (Manhattan, Kans., Crops Res. Div., Agric. Res. Serv., U.S. Dept. of Agric., and Dept. of Flour and Feed Milling Industr., Kansas Agric. Exp. Stat.) Cereal Chem. 40, 658-665 (1963).

Der Einfluß der Zusätze von Glycin, Lysin und Glutaminsäure allein oder in Mischungen mit 17 verschiedenen Zuckern auf Volumen und Färbung von. Krume und Kruste beim Herstellen von Weißbrot wird beschrieben.

Auf die Höhe des Brotvolumens wirkte insbesondere Lysin mindernd, Glutaminsäure eher steigernd. Zucker in Mengen bis zu 4\% (auf Mehl berechnet) ließen, sofern sie vergärbar waren, eine Erhöhung des Brotvolumens erkennen. Ribose als Zutat bei der Brotherstellung bewirkte eine Gelbfärbung der Krume. Eine Verstärkung der Krustenfärbung wurde hauptsächlich bei Einsatz von Raffinose und Pentosen beobachtet. Von den Aminosäuren wurde die Krustenfärbung vorwiegend bei Zusatz von Lysin und Glycin vertieft.

E. Drews (Berlin) 